\title{
The Speculation on the Quantum Mechanics Proceeding From a Principle of the Conservation of Entropy at an Atomic Scale
}

\author{
Khoroshavtsev Y. E. \\ Correspondence: Khoroshavtsev Y. E., Faculty of Automatic Control Systems, State University of Civil Aviation, St. \\ Petersburg, Russia. E-mail: khoroshavtsev@ mail.ru
}

Received: March 17, 2019 Accepted: May 5, 2019 Online Published: May 8, 2019

doi:10.5539/jmr.v11n3p34

URL: https://doi.org/10.5539/jmr.v11n3p34

\begin{abstract}
The entropy approach to a description of quantum processes, represented like the Markov chain, is elaborated. It is supposed that at an atomic scale the law of the conservation of entropy holds. From this principle issues the simple explanation of the different behavior of bosons and fermions in experiments with scattering particles, proved by Pauli. Introducing in consideration Markov' chain, applied to a motion of particles, made possible to write an equation, determining the change of their states. This pure logical result, completed by physical laws, brought to a new equation, generalizing Schrödinger one. It includes electric and magnetic fields as well as the spin of particles. Analysis of its solution for a case of only electric field, led to the formula of tunnel effect. Consideration of the motion of a particle in magnetic field gave the fundamental expression of Lorentz force without any mention of electrodynamics. Addition of an angular momentum in the reasoning by including Hamiltonian, allowed deducing the result, which coincides with the spherically symmetric Schrödinger equation for hydrogen atom. In general case there is the system of equations. If the atom is put in a weak magnetic field, without a possibility of flipping, the classical formulas of Bohr radius orbits and Rydberg energy obtained. The property of intrinsic quantization of electron spin $\hbar / 2$ is explained easily as a solution of the proposed system. These equations do not repeat the known arguments only, but can predict the new phenomena. They describe the effect of spin depolarization of electrons in a strong magnetic field, earlier considered been related to vacuum polarization. The obtained solution gives its magnetic induction equal about $10^{12} \mathrm{Gs}$, that corresponds well with experimental data.
\end{abstract}

Keywords: entropy, quantum mechanics, probability, spin, wave function

\section{Introduction}

The evolution of the quantum mechanics is well known. In December 1900 Max Planck exposed his quantum theory of radiation. Later Bohr, Sommerfeld, De Broglie and others (Feynman, 1963), (Wichmann, 1973), (Schiff, 1949), (Landau, 1977) developed the quantum idea and extended the wave - particle duality from light to all corpuscles. A new stage of the theory started from Schrödinger and Heisenberg equations (Feynman, 1963), (Schiff, 1949), (Messiah, 1966), (Landau, 1977) in which the Hamiltonian (the operator corresponding to a total energy of the system) generates its time evolution. Later subsequently, Dirac's equation, which unifies matrix and wave mechanics, was added (Dirac, 1978), (Schiff, 1949), (Landau, 1977). These equations describe well ones physical phenomenon, but did not manage the others. Therefore, different modifications were elaborated. So, to include the magnetic field into Schrödinger's equation (i.e. Hamiltonian) many methods were suggested. Since a nascence of the theory some of them now are well known and entered in the books (Feynman, 1963), (Wichmann, 1973), (Schiff, 1949), (Messiah, 1966), (Landau, 1977), the others are new and often refer to digital calculations, for instance, by using either the difference time method (Sudiarta, 2008), either a principle of minimal substitution (Arioli, 2003), or addition special polynomial functions (Hitler, 2017) and so on.

Notwithstanding, to understand the strange atom's world the old approaches and ideas, for example, using the semi classical Bohr model of atoms and the notion of De Broglie's waves, remain (Allmendinger, 2016). On the principles of the statistical physics Felix Bloch built his theory of the magnetic resonance that is actual (Slichter, 1992). Each model describes different sides of the problem and has a right of existence ever since.

Later it became clear, that the most accurate solutions are being given by the quantum electrodynamics (Feynman, 1985). But its calculating schemes are more similar to algorithms of the computing mathematics and their results are expressed by numbers (not functions), that obstruct the establishment and understanding of patterns. Although, namely the formulas determine universal laws and constitute a foundation of the theoretical physics. They are endowed with the beauty and harmony, on their basis new phenomenons are predicted and new theories are created. So, the big interest remains to find 
some equation, which the others could be deduced from.

Schrödinger's and Heisenberg's equations were obtained originally in the frame of Hamilton's mechanics (Feynman, 1963), (Landau, 1972), (Messiah, 1966), (Schiff, 1949). In the present investigation a new way is chosen and the main assumptions are taken from the statistical physics and fundamental Planck's law of the quantization of energy. The purpose - is to obtain a generalized quantum equation for the complex wave function $\psi$, proceeding from a hypothetic law of conservation of entropy in Markov systems.

The supposition of information conservation in quantum physics is not new (Qi-Ren Zhang, 2009), (Roncaglia, 2017). The principle of conservation was suggested in different forms either as a claim that an informational content of isolated systems does not change in time, or a unitarity of quantum mechanics prohibits information destruction (Roncaglia, 2017). However, the eminent practical issues on this basis weren't obtained.

The concept of entropy at first was introduced for a description of thermodynamic processes. Subsequently, apart from the essential definition as a ratio of a variation of heat quantity to a temperature of adiabatic system, it got a second one - a logarithm of probabilities of system's states. The first definition is applied to macro systems with a large number of states, commeasurable to Avogadro's number, a second one - when this number is little. In statistical physics the both definitions are connected by the fundamental relationship of thermodynamics, established by Boltzmann (Reif, 1974). Let examine shortly its main postulates.

For adiabatic processes a notion of reversible cycle, when entropy is conserved after a return to an initial state, merited an important theoretical significance and led to the definition of ideal gas and Carnot cycle. In consequence of the real processes are irreversible, an entropy always increases according to Second law of thermodynamics. Mathematically this property is confined in the class of real numbers and is applying to macro systems, but do not involve complex values. However, what will it be in an atomic level when statistical entropy can be calculated on all states, and with an assumption, that a hypothetic law of the conservation of entropy holds? These states only must obey to an exigency to be Markov's (Mansuripur, 1987), (Khoroshavtsev, 2005) (generally, the return to the initial state in Markov chain is analogous to having occurred all Carnot cycle). The answer to this question constitutes a purpose of the present examination.

\section{The Law of Entropy Conservation}

\subsection{Statistical Entropy in the Theory of Information}

The notion of statistical entropy is used also in the theory of information, where it serves as a measure of an uncertainty of random states or events. These events are described by a priory probabilities, taking place before an observation (getting an informational signal through a channel), as well as by posterior ones after an event has occurred (Sheridan, 1975). Probabilities are linked according to Bayes' theorem. In order to the posterior probability takes a value equal a unit versus an initial a priori value, it must be transmitted through the channel from input $x$ to an output $y$ an amount of information

where entropy $\mathrm{H}$, been determined on a host $z$, is given as

$$
T(x, y)=H(x)+H(y)-H(x, y),
$$

$H(z)=-\sum P\left(z_{k}\right) \log P\left(z_{k}\right)$.
Here $P\left(z_{k}\right)$ - is the probability of appearance of a rate $z_{k}, k \underline{\underline{k}} 1, \ldots, n$.

The mathematical methods of the theory of information are well developed and can be borrowed for further investigations.

Let the transmission of information is carried out by a not ideal communication channel in which the transmitted signal can take $n$-values. The conclusion about a true value of input $x$ can be made only statistically by an estimate $y$. A relationship between $x$ and $y$ is described by a matrix of probabilities, which in this case has a dimension $n \times n$. This channel is considered like a black box and it is necessary to evaluate statistically not observable $n$ states $x$ by means of $n$ states $y$. Such formulation has a quantum mechanics analogy: the principle of cause - effect relationships is substituted by principle of uncertainty and probabilities of states.

An action of a channel is described by the transition probabilities matrix. If a channel is ideal, in which an output signal $y$ reproduces exactly an input $x$, then an entropy $H(x)=H(y)=H(x, y)$. The transmitted $T(x, y)$ is equal to $H(x)$ and a priory uncertainty is taken off. After an observation it does not remain an initial uncertainty about a true value of $x$, so the matrix of conditional probabilities is equal to unit $\mathbf{E}$. In case of not ideal channel, there is a loose of information, $T(x, y)<H(x)$ and a knowledge about $x$ can be only suppositional. The conditional probability matrix ceases to be unit.

Let there is a scheme with series of channels, constituting a Markov chain. For Markov processes the matrix of such connection is equal to a product of all matrices. It seems of particular interest the connection of two channels, where in the first - information gets lost, and the second is the recovering one; i.e. generally, both channels are equivalently to 
ideal one. It can be demonstrated, that if in the first matrix of conditional probabilities its elements are nonnegative numbers (according to the classical definition), then at least one element in the second matrix must be negative (Khoroshavtsev, 1999). This result, banning the restitution of signals (without informational redundancy) in the theory of communication, will be useful in the present research.

\subsection{The Principle of the Entropy Conservation in Quantum Mechanics}

A notion of channel can be extended to all devices, which change the states of physical process during an experiment. For example, let us consider the Stern - Gerlach apparatus, which sorts atoms with spin 1 into an inhomogeneous magnetic field.

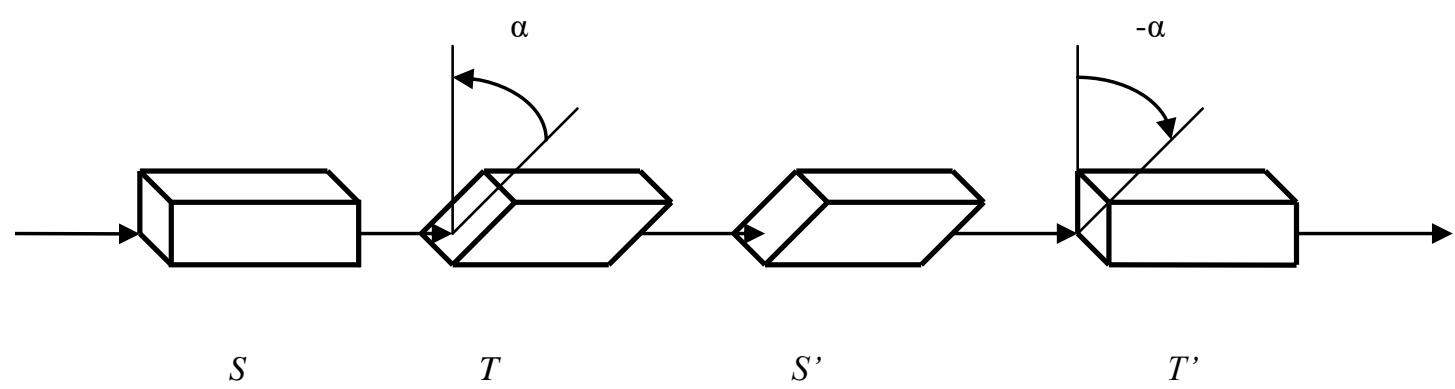

Figure 1. Return to the initial state in an experiment with two Stern - Gerlach apparatus $(S, T)$ and $\left(S^{\prime} T^{\prime}\right)$

There are two apparatus connected in series: $(\mathrm{S}, T)$ and $\left(S^{\prime}, T^{\prime}\right)$. In the first apparatus $T$ is turned at an angle $\alpha$ with respect to $S$, and in the second, $T$ ' turned at - $\alpha$ (Figure 1). The result of such connection is the return to an initial state. It is interesting to check whether entropy is being conserved like at Carnot cycle that means to verify the hypothetic law of conservation of entropy at atom's scale. To do that it is enough to verify if the matrix of series connection of apparatus is equal to unit. For three basic states, the square matrices of conditional probabilities $\mathbf{A}_{1}(\alpha)$ and $\mathbf{A}_{2}(\alpha)$ are expressed as (Feynman, 1963)

$$
\begin{gathered}
\mathbf{A}_{1}(\alpha)=\left\|\begin{array}{ccc}
1+\cos \alpha / 2 & -\sin \alpha / \sqrt{2} & 1-\cos \alpha / 2 \\
\sin \alpha / \sqrt{2} & \cos \alpha & -\sin \alpha / \sqrt{2} \\
1-\cos \alpha / 2 & \sin \alpha / \sqrt{2} & 1+\cos \alpha / 2
\end{array}\right\|, \\
\mathbf{A}_{2}(\alpha)=\left\|\begin{array}{ccc}
1+\cos \alpha / 2 & \sin \alpha / \sqrt{2} & 1-\cos \alpha / 2 \\
-\sin \alpha / \sqrt{2} & \cos \alpha & \sin \alpha / \sqrt{2} \\
1-\cos \alpha / 2 & -\sin \alpha / \sqrt{2} & 1+\cos \alpha / 2
\end{array}\right\|,
\end{gathered}
$$

and it is easy to show that $\mathbf{A}_{1} \mathbf{A}_{2}=\mathbf{E}$. So, entropy is conserved (Khoroshavtsev, 2005).

This result means that the matrix $\mathbf{A}_{2}$ occurs equal inverse to $\mathbf{A}_{1}$, i.e. $\mathbf{A}_{2}(\alpha)=\mathbf{A}_{1}^{-1}(\alpha)$.

On the other hand, because the return to an initial state happens, hence

$$
\mathbf{A}_{1}(\alpha)=\mathbf{A}_{2}(-\alpha) .
$$

In general case, from assumption of the principle of conservation of entropy in atom scale, an important equality follows

$$
\mathbf{A}^{-1}(x)=\mathbf{A}(-x)
$$

where $x$ - a variable of a task.

These matrices can be considered formally as a description of some linear nonsingular transformation. It is known, that the eigen numbers of matrices do not depend on a choice of a basis. According that, it is assumed that $\mathbf{A}(x)$ theoretically can be described in the basis, coinciding with its eigen vectors. In this case, the matrix takes a diagonal form 


$$
\mathbf{A}(x)=\left\|\begin{array}{cccc}
a_{1}(x) & 0 & \ldots & 0 \\
0 & a_{2}(x) & \ldots & 0 \\
. & \cdot & \ldots & . \\
0 & 0 & \ldots & a_{n}(x)
\end{array}\right\|
$$

with the determinant $\Delta=a_{1}(x) a_{2}(x) \ldots a_{n}(x)$, which must be equal to a unity from the condition of probabilities normalizing. So, an inverse matrix can be written as

$$
\mathbf{A}^{-1}=\frac{1}{\Delta}\left\|\begin{array}{cccc}
a_{2} a_{3} \ldots a_{n} & 0 & \ldots & 0 \\
0 & a_{1} a_{3} \ldots a_{n} & \ldots & 0 \\
. & . & \ldots & . \\
0 & 0 & \ldots & a_{1} a_{2} \ldots a_{n-1}
\end{array}\right\|=\left\|\begin{array}{cccc}
a_{1}^{-1} & 0 & \ldots & 0 \\
0 & a_{2}^{-1} & \ldots & 0 \\
. & . & \ldots & . \\
0 & 0 & \ldots & a_{n}^{-1}
\end{array}\right\| .
$$

Comparing (2) and (3), and taking in consideration (1), it can be concluded, that

$$
a_{1}^{-1}(x)=a_{1}(-x), \quad \ldots, \quad a_{n}^{-1}(x)=a_{n}(-x) .
$$

These equalities can be possible only for exponential functions. But in the class of real numbers they cannot be negative that is necessary to a composition of recovering channel if the conservation of entropy holds. Hence, from the principle of conservation of entropy, the main conclusion follows: in the eigen basis elements of matrices of transition probabilities must be imaginary exponents. For even dimension $n$ there are pairs of functions in form $\exp ( \pm i x)$, for odd's - an unity is added as $\exp (0)$. For example, into the eigen basis a matrix $\mathbf{A}_{1}$ transforms in

$$
\mathbf{A}_{1}=\left\|\begin{array}{ccc}
\exp (i \alpha) & 0 & 0 \\
0 & 1 & 0 \\
0 & 0 & \exp (-i \alpha)
\end{array}\right\|, \quad i=\sqrt{-1} .
$$

In concordance to the admitted terminology, these complex values are called probability amplitudes (Feynman, 1963). In general case, a behavior of channels is not described in an eigen basis, respectively, elements of matrices can be any complex numbers. This result partly coincides with the definition of unitary operator in physics (Landau, 1972), but it is not postulated and issues from the hypothetic law of entropy conservation.

A notion of a channel, linking input and output states, can be applied to solving some physical problems, for instance, a diffraction of electrons passing through a slit in a sample. This experiment can be explained using two channels: one (source, sample) and second - (sample, screen) (Figure 2).

The first channel is described with matrix - rows, the second one - columns. A product of matrices is a complex number $F$, its module shows a relative density of hits in a point $x$.

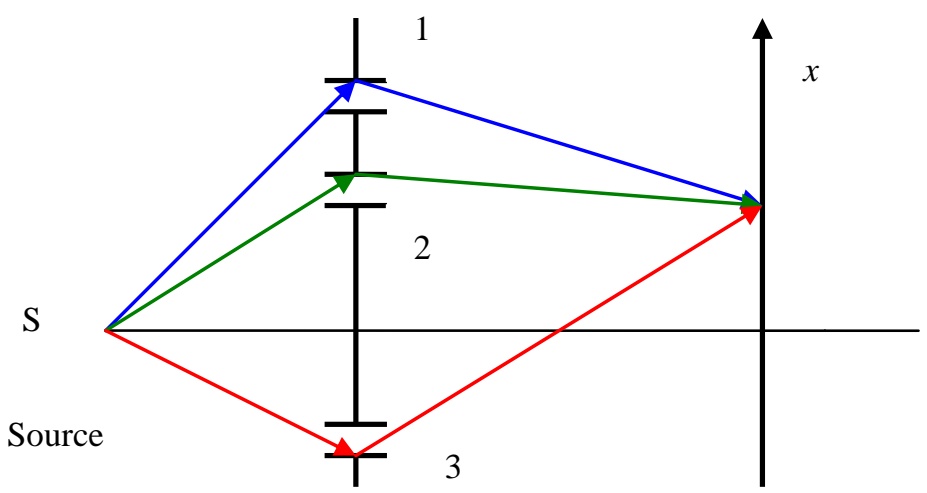

Sample

Screen

Figure 2. An experiment with a diffraction of electrons 


$$
\begin{aligned}
F= & \left\|a_{1} e^{i \alpha_{1}} \quad a_{2} e^{i \alpha_{2}} \quad a_{3} e^{i \alpha_{3}}\right\| \bullet\left\|b_{1} e^{i \beta_{1}} \quad b_{2} e^{i \beta_{2}} \quad b_{3} e^{i \beta_{3}}\right\|^{T}= \\
= & a_{1} b_{1} e^{i\left(\alpha_{1}+\beta_{1}\right)}+a_{2} b_{2} e^{i\left(\alpha_{2}+\beta_{2}\right)}+a_{3} b_{3} e^{i\left(\alpha_{3}+\beta_{3}\right)},
\end{aligned}
$$

where $a, b, \alpha, \beta$ - parameters of an experiment,

$T$ - a symbol of transpose.

The result of multiplication illustrates an interference of probability amplitudes. If it is known, that an electron, for example, passes through a slit 1 , then there is not an uncertainty of states (channels ideal) and matrices become pseudo unit with exactitude of an outputting factor $a_{1} b_{1}$. Hence (4) transforms into

$$
\|1 \quad 0 \quad 0\| \bullet\|1 \quad 0 \quad 0\|^{T}=1
$$

As consequence, interference disappears.

The complex character of $F$ leads to a pure quantum phenomenon, thus by closing one or two slits a number of scintillations on a screen in a point $x$ can increase (Feynman, 1963). Mathematically it follows from (4).

Let us examine a scattering of particles. An experiment consists in calculating of a probability $F(\beta)^{2}$ of scattering of identical particles with a spin $1 / 2$, polarized along axis $z$, in detector $D$, lying on a plane $x y$ (Figure 3 ).

A scheme of experiment can be represented by Markov chain of three channels with their matrices of conditional probabilities $\mathbf{A}_{\mathrm{s}}, \mathbf{R}_{\mathrm{z}}$, and $\mathbf{D}$.
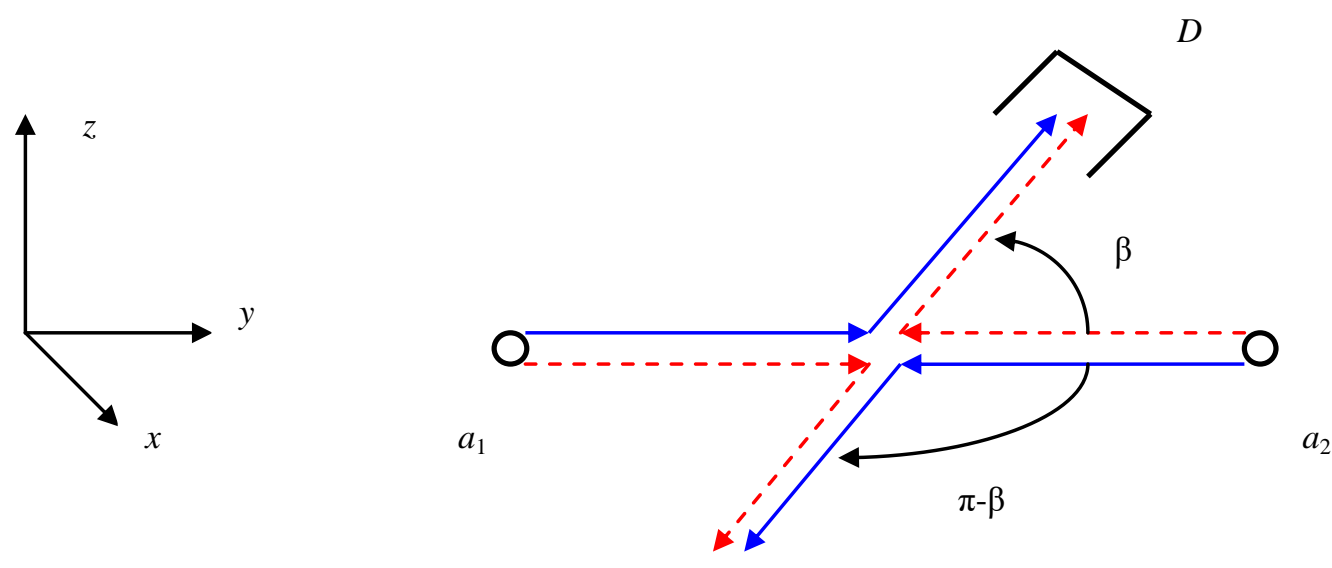

Figure 3. A scattering of identical particles $a_{1}$ and $a_{2}$ having a spin $1 / 2$ in center of mass system

Matrix-rows $\mathbf{A}_{\mathrm{s}}$ of elastic collision which creates an initial uncertainty, when particles can exchange of their individualities, is

$$
\mathbf{A}_{s}=\left\|a_{11} \quad a_{12}\right\|
$$

A square matrix $\mathbf{R}_{\mathrm{z}}$ describes a turn of the spin around axis $z$.

$$
\mathbf{R}_{z}=\left\|\begin{array}{ll}
r_{11} & r_{12} \\
r_{21} & r_{22}
\end{array}\right\|, \quad \mathbf{D}=\left\|\begin{array}{l}
d_{11} \\
d_{21}
\end{array}\right\| .
$$


Matrix-columns D determines a scattering of particles $a_{1}$ or $a_{2}$ in detector.

The probability amplitude to register any particle into detector is given by a matrix product

$$
F(\beta)=\mathbf{A}_{s} \mathbf{R}_{z} \mathbf{D}=\left\|\begin{array}{ll}
a_{11} & a_{12}
\end{array}\right\| \bullet\left\|\begin{array}{ll}
r_{11} & r_{12} \\
r_{21} & r_{22}
\end{array}\right\| \bullet\left\|\begin{array}{l}
d_{11} \\
d_{21}
\end{array}\right\| .
$$

Because the particles are identical, from the symmetry it follows $a_{11}=a_{12}=a$.

The scattering of particles accompanies with a turn of spin at a corresponding angle $\beta$. The matrix $\mathbf{R}_{\mathrm{z}}$ determines this turn around an axis $z$ of a particle, moving along axis $y$. Its elements are known: $r_{12}=r_{21}=0$ and $r_{11}=\exp (i \beta), r_{22}=\exp (-i(\pi-\beta))$, with a little appreciation (Feynman, 1963). Because there are two particles and not one, it is necessary to take a total value of $\beta$, not a half. The twofold angle with respect to (Feynman, 1963) is explained by such a reason. In frontal collision particles scatter in strong opposite directions $+y$ and $-y$, so phases of probability amplitudes must differ by $\pi$. As a consequence,

$$
\mathbf{R}_{z}=\left\|\begin{array}{cc}
e^{i \beta} & 0 \\
0 & e^{-i(\pi-\beta)}
\end{array}\right\|=e^{i \beta \|}\left\|\begin{array}{cc}
1 & 0 \\
0 & -1
\end{array}\right\|, \quad i=\sqrt{-1}
$$

Elements $d_{11}$ and $d_{21}$ of matrix $\mathbf{D}$ determine amplitudes to scatter in a chosen directions. In a center of mass system they will be denoted as $d_{11}=f(\beta), d_{21}=f(\pi-\beta)$ (Feynman, 1963).

Substituting so determined matrices in (5) gives

$$
F(\beta)=a e^{i \beta}[f(\beta)-f(\pi-\beta)]
$$

If a corpuscle is composed of $n$ identical particles, its turn means the turns of each spin that is described by a product of $n$ identical matrices $\mathbf{R}_{z}$, equivalent to a turn of one particle with a spin $1 / 2$ at an angle $n \pi$ or $-n(\pi-\beta)$. Hence,

$$
F(\beta)=a e^{i n \beta}\left[f(\beta)+(-1)^{n} f(\pi-\beta)\right] .
$$

If $n$ is an even number, then according to (6), there is an interference of amplitudes with a sign plus, in case of an odd number a sign will be minus. An even number corresponds to an integer spin of compose corpuscle (boson), an odd number - to a half integer's (fermion). The concept of channel let easily to explain a known fact, proved by Pauli (Landau, 1977) - in scattering experiment bosons interfere in a phase, fermions interfere in a counterphase.

\subsection{Equation of Transition of a Particle From one Special State to Another in Markov Chain}

The concept of Markov chain can be applied to exam a moving particle when entropy is conserved.

Let there is a particle moving along an axis $x$ and having a spin, which can take two possible values: up (+) or down (-). Graph of its states is shown in a Figure 4.

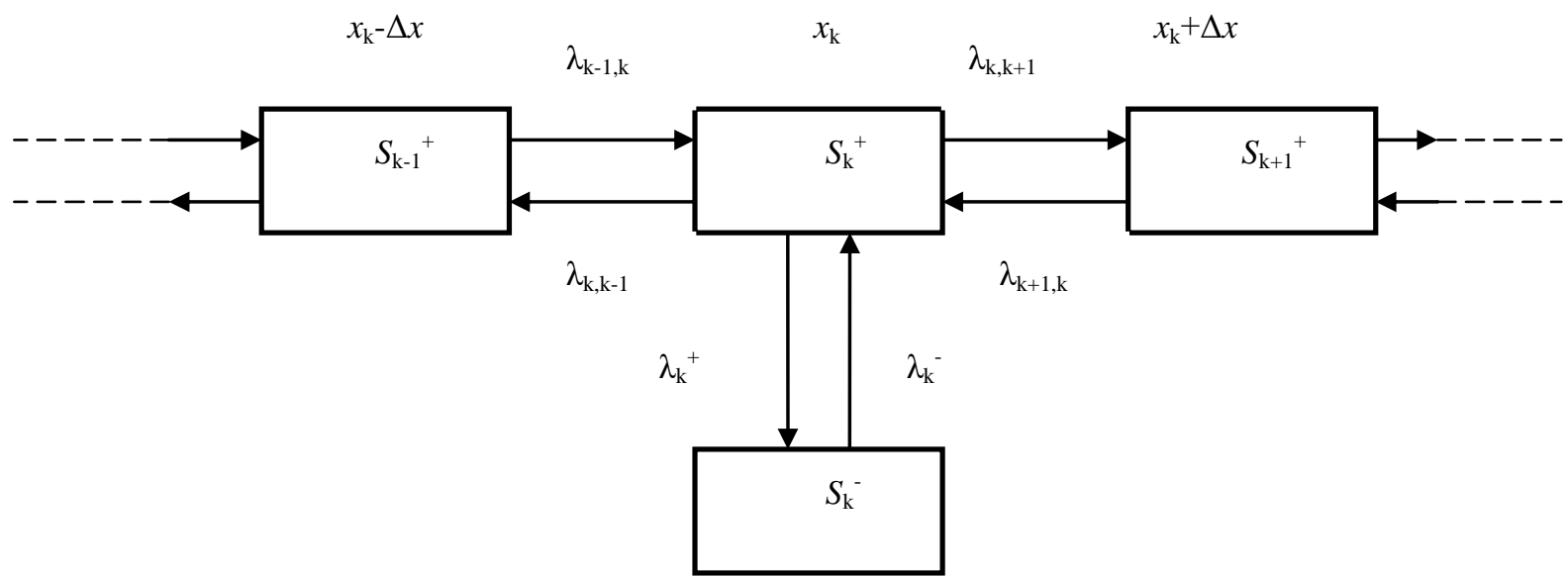

Figure 4. Graph of states of a moving particle with spin 
State $S_{\mathrm{k}}{ }^{+}$corresponds to a location of a particle in the point with a coordinate $x_{\mathrm{k}}$ and a spin + . State $S_{\mathrm{k}}{ }^{-}$is the same, but with a spin -. States $S_{\mathrm{k}-1}{ }^{+}$and $S_{\mathrm{k}+1}{ }^{-}$correspond to locations in points $x_{\mathrm{k}}-\Delta x$ and $x_{\mathrm{k}}+\Delta x$. Intensities $\lambda$ mean an average frequencies of transitions between neighboring states when a particle is exposed to exertion of electromagnetic field. If an external field is absent, it is supposed that all $\lambda$ are equal to each other.

At first, let consider a simplest case of the particle without a field and without a spin, then it remains only states $S_{\mathrm{k}}$ and $S_{\mathrm{k} \pm 1}$ in a line. For Markov processes is impossible either skipping states or a simultaneous appearance of two events.

According to these properties, the possibility that the particle at time $t+\Delta t$ will turn out in a state $S_{\mathrm{k}}$ can occur by three independent ways: - at a time $t$ it just was in $S_{\mathrm{k}}$ and during $\Delta t$ did not transit neither in $S_{\mathrm{k}-1}$ nor in $S_{\mathrm{k}+1}$; - at $t$ it was in $S_{\mathrm{k}-1}$ and during $\Delta t$ transited in $S_{\mathrm{k}}$; - similarly with $S_{\mathrm{k}+1}$. For Markov processes a probability that one event can occur in a span $\Delta t$ is $P(\Delta t)=\lambda \Delta t$. Hence, summarizing possibilities gives

$$
P_{k}(t+\Delta t)=P_{k}(t)(1-\lambda \Delta t)^{2}+P_{k-1}(t) \lambda \Delta t+P_{k+1}(t) \lambda \Delta t
$$

By ignoring the second order terms after simple rearrangement this expression can be rewritten as

$$
\frac{P_{k}(t+\Delta t)-P_{k}(t)}{\Delta t}=\lambda\left[-2 P_{k}(t)+P_{k-1}(t)+P_{k+1}(t)\right]
$$

In a limited case $\Delta t \rightarrow 0$ a differential equation follows

$$
\frac{\partial P\left(x_{k}\right)}{\partial t}=\lambda\left[-2 P\left(x_{k}\right)+P\left(x_{k}-\Delta x\right)+P\left(x_{k}+\Delta x\right)\right] .
$$

Decaying functions $P\left(x_{\mathrm{k}} \pm \Delta x\right)$ in Taylor series and ignoring high order terms yields an equality

$$
\frac{\partial P}{\partial t}=\lambda \Delta x^{2} \frac{\partial^{2} P}{\partial x^{2}}
$$

If to solve this equation from any determined origin position of a particle, its initial state will be described by probability's unit vector. In order to complex probabilities could appear after a while, that is necessary from the claim of entropy conservation, $\lambda$ must be an imaginary number. So, $\lambda=i v, \quad v \in R$. To stress a complex character of probability, a symbol $P$ is being changed on $\psi$ :

$$
i \frac{\partial \psi}{\partial t}+v \Delta x^{2} \frac{\partial^{2} \psi}{\partial x^{2}}=0
$$

This equation predicts transition probabilities of spacial states in Markov chain. Physically, a particle changes a position depending on its energy and momentum. According to the fundamental formulas of quantum physics, uniting wave and corpuscular properties of elementary particles, an energy $E$ and momentum $p$ are determined by

$$
E=h v, \quad p=h / \Delta x .
$$

Here $h$ - Planck constant.

At absence of a field, $E$ - kinetic energy, equal to $p v / 2, p=m v$, where $m, v$ - mass and velocity of a particle. Substituting (8) in (7) leads to

$$
i \frac{\partial \psi}{\partial t}+\frac{h}{2 m} \frac{\partial^{2} \psi}{\partial x^{2}}=0
$$

This is non - relativistic Schrödinger equation for free particle.

\section{The Generalized Quantum Equation}

\subsection{Equation of a Moving Particle in the Electromagnetic Field}

An external field makes the states more or less probable, that influences on $\lambda$. For the static field by a linear approximation intensities $\lambda$ can be represented as

$$
\lambda_{k+1, k}=\lambda_{k}+\left.\frac{\partial \lambda}{\partial x}\right|_{x=x_{k}} \Delta x, \quad \lambda_{k-1, k}=\lambda_{k}-\left.\frac{\partial \lambda}{\partial x}\right|_{x=x_{k}} \Delta x,\left.\quad \frac{\partial \lambda}{\partial x}\right|_{x=x_{k}}=\gamma_{k}
$$


It is admitted from a symmetry $\lambda_{\mathrm{k}, \mathrm{k}+1}=\lambda_{\mathrm{k}, \mathrm{k}-1}=\lambda_{\mathrm{k}}$, with $\lambda_{\mathrm{k}}=i v$ according to (7).

Following the logic of reasoning like a recapitulation of (7), yields an equation of a moving particle in an external field, where intensities $\gamma_{\mathrm{k}}=i \chi$.

$$
i \frac{\partial \psi}{\partial t}+v \Delta x^{2} \frac{\partial^{2} \psi}{\partial x^{2}}+2 \chi \Delta x^{2} \frac{\partial \psi}{\partial x}=0 .
$$

It is assumed, that in electromagnetic field with scalar $\varphi_{\mathrm{e}}$ and vector $\mathbf{A}$ potentials for a particle with a charge $q$, such determination holds

$$
\chi \Delta x^{2}=\frac{q}{m} \sqrt{\frac{\varphi_{e}^{2}}{c^{2}}-\mathbf{A}^{2}} .
$$

Here $c$ - velocity of light.

This is the simplest hypothetic expression with a demanded physical measurement and also Lorentz invariant of an electromagnetic field.

Inserting it in (9) and extending to 3D space leads to an equation

$$
i \frac{\partial \psi}{\partial t}+\frac{h}{2 m} \nabla^{2} \psi+\frac{2 q}{m} \sqrt{\frac{\varphi_{e}^{2}}{c^{2}}-\mathbf{A}^{2}} \nabla \psi=0
$$

It is admitted, that a vector of velocity $\mathbf{v}$ of a particle is parallel to $\operatorname{grad} \varphi_{\mathrm{e}}$ and $\mathbf{A}$.

In one dimension case it takes a view

$$
i \frac{\partial \psi}{\partial t}+\frac{h}{2 m} \frac{\partial^{2} \psi}{\partial^{2} x}+\frac{2 q}{m} \sqrt{\frac{\varphi_{e}^{2}}{c^{2}}-\mathbf{A}^{2}} \frac{\partial \psi}{\partial x}=0 .
$$

It can be easily demonstrated by substitution, that the function $\psi(x, t)=\exp (k x+i \omega t)$,

where $\omega=k v$ and $k=\frac{2}{h}\left(m v-2 q \sqrt{\frac{\varphi_{e}^{2}}{c^{2}}-\mathbf{A}^{2}}\right)$,

satisfies to (11). Let's examine its consequences.

First of all it is interesting to investigate a simplest case when only an electric field with potential $\varphi$ is present.

Without magnetic field $\mathbf{A}=0$ and

$$
k=\frac{2}{h}\left(m v-2 \frac{q \varphi_{e}}{c}\right)=\frac{2}{h c}\left(m v c-2 U_{\varphi}\right),
$$

where $U_{\varphi}=q \varphi_{\mathrm{e}}-$ a potential energy of a charge.

Because a total energy $E_{\Sigma}=m v c$ and kinetic one can be expressed as $E=E_{\Sigma}-U_{\varphi}$, the solution of (11) in electrical field takes a view

$$
\psi(x, t)=\exp \left[\frac{2}{h c}\left(E-U_{\varphi}\right)(x+i v t)\right] .
$$

There is a non zero probability $P=\psi^{2}$ for a particle to tunnel through a potential barrier even if $E<U_{\varphi}$. Now, let there is a magnetic field $\mathbf{A}$ and $\varphi_{\mathrm{e}}=0$.

In a magnetic field the trajectory of a charge curves. Let there is a circular revolving with constant radius $r$. Then coordinate $r \varphi_{\mathrm{k}}$ corresponds to the state $S_{\mathrm{k}}$, where $\varphi$ - an azimuth angle (Figure 5). So, an equation (11) transforms into

$$
i\left(\frac{\partial \psi}{\partial t}+\frac{q A}{m r} \frac{\partial \psi}{\partial \varphi}\right)+\frac{\hbar}{2 m r^{2}} \frac{\partial^{2} \psi}{\partial \varphi^{2}}=0 \quad, \quad r=\mathrm{const} .
$$




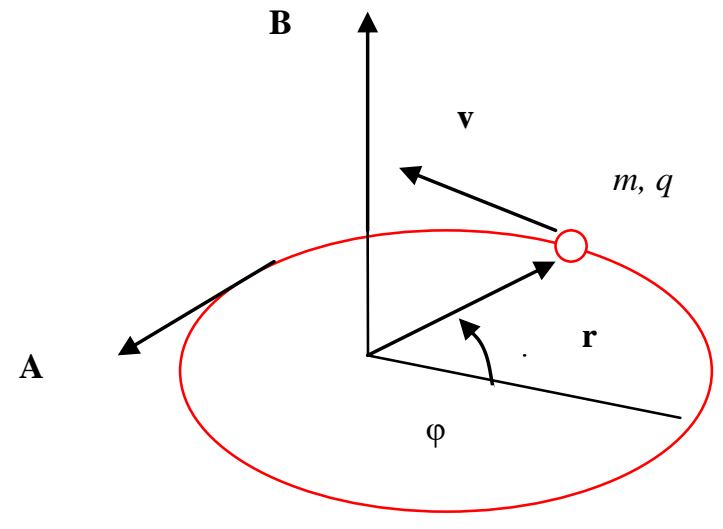

Figure 5. The trajectory of a charge in magnetic field

Its solution is searched as a function $\psi(\varphi, t)=\exp [i(k r \varphi-\omega t)]$, with frequency $\omega=k v$, and wave number $k$. Inserting $\psi$ in $(12)$ yields $k=2(m v-2 q A) / \hbar$.

For the circular contour from symmetry a position of a particle in any point of an orbit is equally likely, i.e. $\psi \neq \psi(\varphi)$. That is possible only if $k=0$, hence, $m v=2 q A$.

In a homogenous magnetic field with an induction $\mathbf{B}$ according to Stokes theorem an equality $A=B r / 2$ holds, where $A, B, r$, $v$ - modules of vectors in Figure 5, Thus, such result follows: $m v=q B r$. It can be rewritten in a known view $m v^{2} / r=q B v$.

This solution expresses a classical condition, namely, a centrifugal force is equal to Lorentz one. It can be said differently, the solution of equation (12) determines a magnetic force, which equilibrates the centrifugal one. The formula of classical physics is obtained from the quantum equation.

\subsection{Spin in the Quantum Equation}

In order to add a spin in equation it is necessary to take in consideration all states in Figure 4 (Khoroshavtsev, 2006). In Markov chain in a span $\Delta t$ can occur only one event, either an overturn of a spin, or a step $\Delta x$. Respectively, at a time $t+\Delta t$ a particle can take a state $S_{\mathrm{k}}{ }^{+}$by four ways: - at time $t$ it was there, and during $\Delta t$ it did not transit neither in $S_{\mathrm{k}}{ }^{-}$, neither in $S_{\mathrm{k}-1}{ }^{+}$, nor in $S_{\mathrm{k}+1}{ }^{+}$; - at $t$ it was in $S_{\mathrm{k}}{ }^{-}$and transited in $S_{\mathrm{k}}{ }^{+} ;$- similarly with $S_{\mathrm{k}-1}{ }^{+}$and $S_{\mathrm{k}+1}{ }^{+}$. The probability of that

$$
P_{k}^{+}(t+\Delta t)=P_{k}^{+}(t)\left(1-\lambda_{k} \Delta t\right)^{2}\left(1-\lambda_{k}^{+} \Delta t\right)+P_{k}^{-}(t) \lambda_{k}^{-} \Delta t+P_{k-1}^{+}(t)\left(\lambda_{k}-\gamma \Delta x\right) \Delta t+P_{k+1}^{+}(t)\left(\lambda_{k}+\gamma \Delta x\right) \Delta t,
$$

where - $\lambda_{k}=\lambda_{k, k+1}=\lambda_{k, k-1} ; \quad \gamma=\left.\frac{\partial \lambda}{\partial x}\right|_{x=x_{k}}$

By analogy, repeating all precedent rearrangements, it can be demonstrated, that

$$
i \frac{\partial \psi^{+}}{\partial t}+\frac{h}{2 m} \frac{\partial^{2} \psi^{+}}{\partial x^{2}}+\frac{2 q}{m} \sqrt{\frac{\varphi_{e}^{2}}{c^{2}}-\mathbf{A}^{2}} \frac{\partial \psi^{+}}{\partial x}=v^{+} \psi^{+}-v^{-} \psi^{-} .
$$

Let there is a motionless particle with a spin in magnetic field. For stiff particle all terms in (13), containing $\partial / \partial x$ and $\partial^{2} / \partial x^{2}$, become equal to zero, so

$$
i \frac{d \psi^{+}}{d t}=v^{+} \psi^{+}-v^{-} \psi^{-} .
$$

Here, there are two unknown functions $\psi^{+}$and $\psi^{-}$, however, one equation. Missing one is obtained by analogy with Figure 4, but built relatively a state $S_{\mathrm{k}}{ }^{-}$similarly. The resulting system can be written in a matrix form

$$
i \frac{d \boldsymbol{\psi}}{d t}=\frac{1}{h} \mathbf{H} \boldsymbol{\psi}, \quad \boldsymbol{\psi}=\left\|\begin{array}{l}
\psi^{+} \\
\psi^{-}
\end{array}\right\|,
$$


where elements of $\mathbf{H}$ - the values of energies, dependent on interaction between a magnetic field and magnetic moment of a particle, staying in two basic orientations.

It is easy to notice, that $\mathbf{H}$-Hamilton matrix in Heisenberg equation (Feynman, 1963), (Landau, 1972). If a magnetic moment directed along a field on $z$, i.e., $\boldsymbol{\mu}=\boldsymbol{\mu}_{z}$, and $\mathbf{B}=\mathbf{B}_{z}$, then a Hamiltonian takes a diagonal form with elements $\pm \mu_{\mathrm{z}} B_{\mathrm{z}}, \mu_{\mathrm{z}}$ - Bohr magneton. Matrix $\mathbf{H}$ becomes expressed in eigen basis, corresponding to stationary states, and (14) breaks up to two independent equations. For a function $\psi^{+}$(for $\psi^{-}-$similarly) that is

$$
i h \frac{d \psi^{+}}{d t}=\mu_{z} B_{z} \psi^{+}
$$

Its solution is well known: $\psi^{+}=C \exp \left(-i \mu_{z} B_{z} t / h\right), C$-constant.

Generalizing (13) and (14) in a case of a moving particle, yields a system

$$
i \frac{\partial \boldsymbol{\psi}}{\partial t}+\frac{h}{2 m} \nabla^{2} \boldsymbol{\psi}+\frac{2 q}{m} \sqrt{\frac{\varphi_{e}^{2}}{c^{2}}-\mathbf{A}^{2}} \nabla \boldsymbol{\psi}=\frac{1}{h} \mathbf{H} \boldsymbol{\psi}, \quad \boldsymbol{\psi}=\left\|\psi_{1} \cdots \psi_{n}\right\|^{T} .
$$

Correspond to early made admissions; vector of speed $\mathbf{v}$ is parallel to grad $\varphi_{\mathrm{e}}$ and to $\mathbf{A}, n$ - number of energetic states in Hamiltonian.

To solve a system (16), it is desirable to write it into eigen basis and get independent equations like (15). Such basis can be guessed from reasoning of symmetry when a correlation between states is absent. In general case an eigen basis is a pure mathematical construction of a linear algebra.

Let consider a charged particle moving into a field of a central force - electron in an atom of hydrogen, which is put in an origin. Its orbital momentum is $\mathbf{J}$ and intrinsic spin ignored. The eigen basis is formed with two symmetric states: momentum 'up' and 'down'. In this basis the right hand side of system (16) looses crossing terms and corresponding diagonal elements of Hamiltonian become equal to double energy of revolving $\mathbf{J} \boldsymbol{\Omega}$. Double - because a flip is accompanied by double changing of momentum $\mathbf{J}$, where $\boldsymbol{\Omega}$-angular speed of a particle.

Because of a spacial symmetry it is likely to pass to spherical coordinates related with Cartesians by transform $x=r \sin \theta \cos \varphi, y=r \sin \theta \sin \varphi, z=r \cos \theta$. Into new coordinates and in eigen basis a solving of (16) consists in solving of two identical equation (the second differs only by a sign of $J$ )

$$
\begin{gathered}
i \frac{\partial \psi}{\partial t}+\frac{\hbar}{2 m} \frac{\partial^{2} \psi}{\partial r^{2}}+\left\{\frac{\hbar}{m r}+\frac{2 q}{m} \sqrt{\frac{\varphi_{e}^{2}}{c^{2}}-\mathbf{A}^{2}}[\sin \theta(\cos \varphi+\sin \varphi)+\cos \theta]\right\} \frac{\partial \psi}{\partial r}+ \\
+\frac{\hbar}{2 m r^{2}} \frac{\partial^{2} \psi}{\partial \theta^{2}}+\left\{\frac{\hbar}{2 m r^{2}} \operatorname{ctg} \theta+\frac{2 q}{m r} \sqrt{\frac{\varphi_{e}^{2}}{c^{2}}-\mathbf{A}^{2}}[\cos \theta(\cos \varphi+\sin \varphi)-\sin \theta]\right\} \frac{\partial \psi}{\partial \theta}- \\
-\frac{\hbar}{2 m r^{2} \sin ^{2} \theta} \frac{\partial^{2} \psi}{\partial \varphi^{2}}+\frac{2 q}{m r \sin \theta} \sqrt{\frac{\varphi_{e}^{2}}{c^{2}}-\mathbf{A}^{2}}(\cos \varphi-\sin \varphi) \frac{\partial \psi}{\partial \varphi}=\frac{1}{\hbar} J \Omega \psi .
\end{gathered}
$$

Let use Bohr's atomic model in which $\mathbf{A}=0$ and with electron, revolving on a circular orbit, so its vector of velocity $\mathbf{v}(t)$ is perpendicular to the gradient of electrical field. This simplifies (17): by symmetry, derivatives with respect to angular coordinates disappear as well as the terms containing the radical. Additionally, from the condition of an equality of centrifuge and electrostatic forces, the energy of angular momentum is expressed as

$$
J \Omega=-e^{2} / r,
$$

where $e$ - elementary charge. So (17) can be rewritten in a form

$$
i \hbar \frac{\partial \psi}{\partial t}+\frac{\hbar}{2 m}\left(\frac{\partial^{2} \psi}{\partial r^{2}}+\frac{2}{r} \frac{\partial \psi}{\partial r}\right)=-\frac{e^{2}}{r} \psi
$$

That is the spherically symmetric Schrödinger equation for hydrogen atom (Feynman, 1963). Its solutions are well known. However, if the atom is put into an external field, it is necessary to solve all system (16) or (17).

It is interesting to analyze a simple motion of a particle with a constant radius $r=R$ and an azimuth angle $\varphi$. Then from (17) 
issues one equation (the second is the similar).

$$
i \frac{\partial \psi}{\partial t}+\frac{\hbar}{2 m R^{2}} \frac{\partial^{2} \psi}{\partial \varphi^{2}}=\frac{1}{\hbar} J \Omega \psi
$$

The wave function $\psi(t, \varphi)=\exp [i(k R \varphi-\omega t)]$ with parameters $k$ and $\omega$ fulfils $(18)$, if $k^{2}=\frac{2 m \Omega}{\hbar^{2}}\left(\frac{\omega}{\Omega} \hbar-J\right)$. It is clear from symmetry, that the solution must not depend on $\varphi$, hence $k=0$ and consequently $J=\frac{\omega}{\Omega} \hbar$.

For a steady rotation an angle $\varphi=\Omega t$, and respectively, the solution can be given as $\psi=\exp [i(k R \Omega-\omega) t]$.

After a period $T=2 \pi / \Omega$ a return to the initial state happens and the phase of a wave function changes on $\pi$ (not $2 \pi$ because a sign of $\psi$ does not matter). Consequently,

$$
\begin{gathered}
(k R \Omega-\omega) \frac{2 \pi}{\Omega}= \pm \pi, \\
k=\frac{1}{R}\left(\frac{\omega}{\Omega} \pm \frac{1}{2}\right) .
\end{gathered}
$$

In order to $k=0$ an equality $\omega / \Omega= \pm 1 / 2$ follows, that leads to the fundamental intrinsic property $J= \pm \hbar / 2$.

Coming back to hydrogen atom- let an electron revolves with speed $\mathbf{v}$, attracted by electric force of a nucleus in a weak homogeny magnetic field $\mathbf{B}$. Vector $\mathbf{B}$ is perpendicularly to an orbit plane $x y$, a flip of momentum is supposed to be forbidden. In polar coordinates with constant orbit radius $r=R$ and azimuth angle $\varphi$, a system (17) transforms in

$$
i \frac{\partial \boldsymbol{\psi}}{\partial t}+\frac{\hbar}{2 m R^{2}} \frac{\partial^{2} \boldsymbol{\psi}}{\partial \varphi^{2}}+\frac{2 q}{m R} \sqrt{\frac{\varphi_{e}^{2}}{c^{2}}-A^{2}} \frac{\partial \boldsymbol{\psi}}{\partial \varphi}=\frac{1}{\hbar} \mathbf{H} \boldsymbol{\psi} .
$$

There are two states $\psi^{+}$and $\psi^{-}$- angular momentum directed along $\pm z$, and Hamiltonian takes a diagonal form

$$
\mathbf{H}=\left\|\begin{array}{cc}
m v^{2} & 0 \\
0 & m v^{2}
\end{array}\right\| .
$$

Its diagonal elements equal to a double kinetic energy, non diagonal ones equal to zero because an overturn is banned. So, (19) breaks up to two identical equations for $\psi$

$$
i\left(\frac{\partial \psi}{\partial t}+\frac{q A}{m R} \frac{\partial \psi}{\partial \varphi}\right)+\frac{\hbar}{2 m R^{2}} \frac{\partial^{2} \psi}{\partial \varphi^{2}}=\frac{1}{\hbar} m v^{2} \psi
$$

Terms, containing $\varphi_{\mathrm{e}}$, are excluded, because $\operatorname{grad} \varphi_{\mathrm{e}}$ is perpendicularly to $\mathbf{v}$, and for this configuration vector $\mathbf{A}$ changed on a scalar A.

The solution of (20) is searched as a function $\psi(\varphi, t)=\exp [i(k R \varphi-\omega t)]$, where wave number $k$ and frequency $\omega-$ parameters of an exponent. Its substitution in (20) leads to a quadratic equation for $k$

$$
k^{2}+2 \frac{q A}{m \hbar} k+2 m\left(\frac{m v^{2}}{\hbar^{2}}-\frac{\omega}{\hbar}\right)=0
$$

with roots

$$
k_{1,2}=-\frac{q A}{m \hbar} \pm \sqrt{\frac{q^{2} A^{2}}{m^{2} \hbar^{2}}-2 m\left(\frac{m v^{2}}{\hbar^{2}}-\frac{\omega}{\hbar}\right)} .
$$

It is evident from a symmetry, the solution must not depend on an angle $\varphi$, so $k=0$. It will happen if $\frac{m v^{2}}{\hbar^{2}}-\frac{\omega}{\hbar}=0$, i.e. 


$$
\omega=\frac{m v^{2}}{\hbar} .
$$

On the other hand, the angular speed of hard spheres revolving electron is constant, hence, $\varphi=\frac{v}{R} t$. Substituting this in $\psi$ yields $\psi=\exp [i(k v-\omega) t]$.

A return to an arbitrary initial state happens when a phase changes on $2 \pi$, that is observed after a period $t=T=2 \pi R / v$. Thus,

$$
(k v-\omega) \frac{2 \pi R}{v}=2 \pi n \quad, \quad n= \pm 1, \pm 2, \ldots
$$

Here $2 \pi$ is taken, because spins of an electron and nucleus do not coincide and a state with $\varphi=\pi$ differs from a state with $2 \pi$. (Figure 6); earlier in (18) electron was considered to be alone. Substituting (21) in (22) gives $k-\frac{m v}{\hbar}=\frac{n}{R}$, that for $k=0$ leads to the relationship between de Broglie wavelength and a radius of Bohr orbit:

$$
\frac{\hbar}{m v}=\frac{R}{n} \text {. }
$$

By means of an equality of electrostatic and centripetal forces $m v^{2} / R=e^{2} / R^{2}$, that could be rewritten as $1 / R=m v^{2} / e^{2}$. This is the known condition of quantization of energy levels. For kinetic energy of electrons

$$
E=\frac{m v^{2}}{2}=\frac{m e^{4}}{2 n^{2} \hbar^{2}}=\frac{E_{R}}{n^{2}},
$$

where $E_{R}-$ Rydberg energy.

The solving is simpler than one, obtained from Schrödinger equation with an admission of a series convergence (Feynman, 1963).

These results are achieved as a solution of proposed quantum equation. To appreciate its value, it is desirable that it predicts new phenomena.

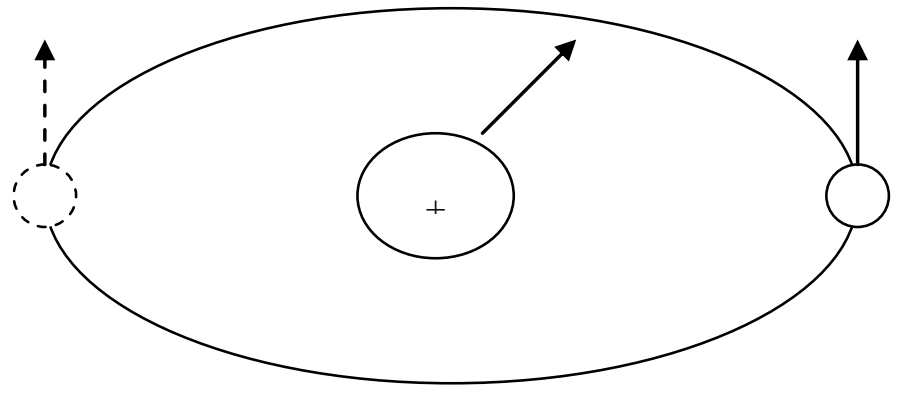

Figure 6. An atom of hydrogen. Arrows show the directions of spins

\section{Spin Depolarization in a Strong Magnetic Field}

On the way to embed a magnetic field in Schrödinger equation many approaches were suggested, for example, by modifying Hamiltonian (Feynman, 1963), (Landau, 1972), or by adding new magnetic and electric states (Kholmetskii, 2017) and so on.

In order to describe a behavior of magnetic momentum into an external field, Bloch elaborated an equation by analogy with a description of precession of a mechanical gyroscope under a moment of forces (Slichter, 1992). But it is a semi classical equation, which does not show anomaly effects. So, it seems of particular interest to apply the hypothetic equation to explain the phenomena of spin depolarization (Khoroshavtsev, 2008). In a strong magnetic field a polarization vanishes (thought because of polarization of vacuum (Ternov, 1986)). 
Let there is an electron gas in magnetic field. Due to chaotic electric interaction the summary force on charge is assumed equal zero. An orbital angular momentum is ignored as well, electrons move in a plane, normal to induction $\mathbf{B}$. Then, there are two distinct states $\psi^{+}$and $\psi^{-}$, which determine directions of an electron magnetic moment, that have a possibility of flipping.

The diagonal elements of Hamiltonian $\mathbf{H}$ describe an energy of electron, non diagonal -energy of interaction between magnetic moment and field, that is $-\mu_{\mathrm{B}} B$, where $\mu_{\mathrm{B}}$ - Bohr magneton. The energy of an electron for the present approximate calculation is admitted as $m c^{2}$ (Ekstrom, 1981).

Hence,

$$
\mathbf{H}=\left\|\begin{array}{cc}
m c^{2} & -\mu_{B} B \\
-\mu_{B} B & m c^{2}
\end{array}\right\|, \quad \mu_{B}=9.27 \cdot 10^{-24}[\mathrm{~J} / \mathrm{T}]
$$

in SI system. Keeping in mind an equality $A=B R / 2$, applied for homogenous field according to Stokes theorem, in polar coordinates (18) transforms in

$$
\left\{\begin{array}{l}
i\left(\frac{\partial \psi^{+}}{\partial t}+\frac{2 e A}{m R} \frac{\partial \psi^{+}}{\partial \varphi}\right)+\frac{\hbar}{2 m R^{2}} \frac{\partial^{2} \psi^{+}}{\partial \varphi^{2}}=\frac{1}{\hbar}\left(h_{11} \psi^{+}+h_{12} \psi^{-}\right) \\
i\left(\frac{\partial \psi^{-}}{\partial t}+\frac{2 e A}{m R} \frac{\partial \psi^{-}}{\partial \varphi}\right)+\frac{\hbar}{2 m R^{2}} \frac{\partial^{2} \psi^{-}}{\partial \varphi^{2}}=\frac{1}{\hbar}\left(h_{21} \psi^{+}+h_{22} \psi^{-}\right)
\end{array}\right.
$$

where $h_{11}, h_{12}, h_{21}, h_{22}$ - elements of $\mathbf{H}$.

Addition of two these equations results to

$$
i\left[\frac{\partial}{\partial t}\left(\psi^{+}+\psi^{-}\right)+\frac{e B}{m} \frac{\partial}{\partial \varphi}\left(\psi^{+}+\psi^{-}\right)\right]+\frac{\hbar}{2 m R^{2}} \frac{\partial^{2}}{\partial \varphi^{2}}\left(\psi^{+}+\psi^{-}\right)=\frac{2}{\hbar}\left(m c^{2}-\mu_{B} B\right)\left(\psi^{+}+\psi^{-}\right) .
$$

By introduction a new function $\psi_{1}=\psi^{+}+\psi^{-}$, the required solution is searched as $\psi_{1}(\varphi, t)=\exp [i(k R \varphi-\omega t)]$, where $\omega=k v$ as usual. After simple rearrangement it issues

$$
\hbar k^{2}-2(m v-e B R) k+\frac{4 m}{\hbar}\left(m c^{2}-\mu_{B} B\right)=0
$$

In order to find $k$ it is necessary to solve (23), in which the factor $m v-e B R$ equals to zero as a sequence of an equality between centrifuge and Lorentz forces.

So, for the direct wave

$$
k=\sqrt{\frac{4 m}{\hbar^{2}}\left(\mu_{B} B-m c^{2}\right)} .
$$

If in (24) the first addend beneath a radical is less than the second one, that occurs when $B<B_{s}$, where $B_{s}=\frac{m c^{2}}{\mu_{B}}$, then $k$ will be an imaginary number and a wave function gets the real factor $\left(i^{2}=-1\right)$, i.e.

$$
\psi_{1}(\varphi, t)=\mathrm{e}^{-|k| R \varphi} \exp (-i \omega t)
$$

Physically this result means, that the magnetic field in the diapason of $0<B<B_{s}$ produces the polarization. However, when $B>B_{s}$ a number $k$ becomes real and consequently $\psi_{1}$ - pure imaginary exponent. Polarization disappears.

The solutions (24) and (25) show also that with an increase of induction $B\left(B<B_{s}\right)$, module of $k$ decreases, hence, $\psi_{1}$ and polarization increase (because of minus before $k$ ). When $B \rightarrow B_{s}$, then $\psi_{1} \rightarrow 1$ exponentially.

The analogous result takes a place about function $\psi_{2}$. The required $\psi^{+}$and $\psi^{-}$are the linear combinations of $\psi_{1}$ and $\psi_{2}$., but they are not interesting here.

It is important to compare a theoretical prediction with experimental data. Inserting physical constants in (24) gives a 
value $B_{s}=8.8 \cdot 10^{8}$ Tesla or $8.8 \cdot 10^{12}$ Gs.

The experiments concerning an influence of strong magnetic fields on the polarization of electrons are usually based on investigations of a cyclotron radiation of plasma (Ternov, 1986). According to (Pavlov, 1979), the depolarization of a radiation of cold plasma $\left(k_{B} T^{\circ}<<m c^{2}, k_{B}\right.$ - Boltzmann constant) occurs in magnetic field with induction $B \geq 4,4 \cdot 10^{13}$ Gs. This effect also is observed in $X$-ray radiation of neutron stars $\left(\mathrm{B} \sim 10^{11} \div 10^{13} \mathrm{Gs}\right.$ ) (Rogerson, 1973). These data sustain the exposed theory.

It needs to mean, the present data analysis is approximate. In Hamiltonian the states, corresponding to acts of radiation, for simplicity are ignored, although namely the radiation lets to observe the polarization. Such assumption can be valid only if energy of interaction of spin with the field more exceeds the energy of quant $E=\hbar \omega$.

For $B_{s} \approx 10^{13} \mathrm{Gs}$ an energy of interaction has an order about $10^{-7} \mathrm{Erg}$, while $X$-ray satellites work in the diapason below 10 $\mathrm{KeV}\left(\approx 10^{-8} \mathrm{Erg}\right)$ and admitted supposition holds. However, more shortwave observations (Kruesi, 2016$)$ do not correspond to the scheme with two states.

\section{Result}

The assumption of the principle of information conservation in quantum mechanics allowed proving that the operator of evolution between states in a model of Markov chain, in general case, must be expressed by matrices of complex elements that are fully correlated with a postulated definition of unitary operator. From this model issues the simple explanation of the different behavior of bosons and fermions in experiments with scattering particles, proved by Pauli.

The model of a change of spacial states of moving particles like Markov chain supplemented by the claim of entropy conservation led to (1- dimension) equation (7) for required complex probabilities (wave function). In order to transform that pure logical result in physical formula the Planck law and Heisenberg uncertainty principle were used. Addition of an angular momentum in the reasoning by including Hamiltonian, allowed deducing the result, which coincides with the spherically symmetric Schrödinger equation for hydrogen atom. In general case, the systems (16) and (17) of equations describing a motion of a particle with a spin in electromagnetic field were obtained.

Analyzing their possible solutions gave many results: the formulas of tunnel effect, Bohr radius orbits, Rydberg energy, quantization by $\hbar / 2$ of a spin. The particular interest represents the solution for the circle motion of an electron. In order to balance the centrifuge force, a magnetic force must exist. It occurs; this force follows from the mathematical solution of the proposed equation and coincides totally with the definition of Lorentz force (magnetic part) without any mention of classical electrodynamics.

The found equation does not repeat the known arguments only, but can predict the new phenomena. It describes the effect of spin depolarization of electrons in a strong magnetic field, thought related to vacuum polarization. The obtained solution shows a magnetic induction equal about $10^{12} \mathrm{Gs}$, that corresponds well with experimental data.

\section{Discussion}

The hypothetic law of conservation of statistical entropy supposes that all physical processes must be discrete. So the space of events must be finite (equal $N$ ), otherwise a normalization of probabilities would be impossible. Discreteness means that between two neighboring states there is not any intermediary one. For example, if in experiment there was a possibility to observe simultaneously the projections of spin on arbitrary directions, then a number of states would be infinite and entropy could not be determined. At any representation spin has only two states - minimal possible number that means the entropy must be minimal according to the law $\ln N$. So in concordance of Boltzmann relationship in thermodynamics the minimum of entropy corresponds to energy minimum. Hence, the principle of entropy conservation transforms in the claim of minimal energy.

In order to determine entropy, the sample space needs to be known. However, at the real experiment always exist non-considered data and consequently new events (Feynman wrote what the universal set is (Feynman, 1963)). Notwithstanding, if to admit that entropy conserves for any Borel set, then any finite sample space is suitable. The other question is how exactly an assumed scheme of an experiment corresponds to the real picture of the world. In probability theory algebraic operations on Borel random events are called sigma algebra. According to its postulates the sample space can be even unobservable, but if to demand that any its subset was complete for algebraic operations (conjuction, inversion...) i.e. were Borel's, this subset can be used as a correct sample space.

In the present investigation on the figure 4 spin at first was ignored, and an expression, which described a tunnel effect as well as Lorentz force, was obtained. The addition of spin yielded an equation, which coincides with Schrödinger one, gave formulas of quantization of orbits and angular momentum, predicted depolarization of electrons in the strong magnetic field. Each time the sample space was modified.

The result in the form of systems (16) or (17) corresponds only to electromagnetic interaction, but can be expanded. The 
proposed entropy approach, completed with Markov chain, may be applied to different computing schemes like shown on figure 4 or probably Feynman diagrams (Feynman, 1985). It gives analytic solutions and let the construction of new equations.

The theoretical achievement consists also in establishment of a relationship between quantum and classical physics by a definition of Lorentz force as a solution of an equation. In particular cases, the found equation gives the same results as Schrödinger one and forecasts novel.

\section{Conclusion}

The present investigation is based on the hypothetic physical law that at an atomic scale the entropy of quantum system conserves. To calculate entropy all the states of system must be discrete, that presents the kernel of quantum physics, and the transition from one state to another can be described statistically using the matrices of probabilities in Markov chain of events. By analogy with a connection of channels in the theory of information and based on the principle of entropy conservation it was demonstrated that these probabilities must be complex numbers or functions $(\psi)$. Applying this model to the moving particles allowed obtaining the generalized quantum equation, including spin, electric and magnetic field. Its solutions show an intrinsic relationship with classical electrodynamics and correspond well with known data.

The obtained equation is Lorentz invariant with respect to electromagnetic field and has a matrix form like Dirac's one, that prove a succession of the theory.

It is probably; the exposed approach is not confined by electromagnetic forces only, but can be applied to strong or weak interactions. But then, the definition of coefficients $v$ and $\chi$ in (9) must stand on the others physical laws.

\section{References}

Allmendinger, T. (2016). A classical approach to the De Broglie-wave based on Bohr's H-Atom-model. International journal of applied mathematics and theoretical physics, 2(1), 1- 15.

Arioli, J., \& Szulkin, A. (2003). A semi linear Schrödinger equation in the presence of a magnetic field. Arch. Rat. Mech. Anal, (170), 277-295. https://doi.org/10.1007/s00205-003-0274-5

Dirac, P. A. M. (1978). Directions in physics. Edited by H. Hora and J. R. Shepanski. John Wiley \& Sons, New York.

Ekstrom, P., \& Wineland, D. J. (1981). Separated electron. Physics - Uspekhi, 134, 711-730. (in Russian). https://doi.org/10.3367/UFNr.0134.198108e.0711

Feynman, R. P. (1985). QED - the strange theory of light and matter. Alix G. Mautner. Memorial lectures. Princeton University Press.

Feynman, R. P., Leighton, R. B., \& Sands, M. (1963). The Feynman lectures on physics. Vol.3. Addison - Wesley publishing company, inc. reading, Massachusetts, Paolo Alto. London.

Hitler, L., Iserom, I. B., Akakuru, O. U., Homas, O. M., Innocent, J., \& Pigweh, A. I. (2017). Radial solution of the S-wave Schrödinger equation with Kratzer plus modified Deng-Fan potential under the framework of Nikiforov-Uvarov method. International journal of applied mathematics and theoretical physics, 3(4), 97-100. https://doi.org/10.11648/j.ijamtp.20170304.14

Kholmetskii, A., \& Yarman, T. (2017). Quantum phases for a charged particle and electric/magnetic dipole in an electromagnetic field. EPL (Europhysics Letters), 120(4). https://doi.org/10.1209/0295-5075/120/40007

Khoroshavtsev, Y. E. (1999). On a problem of composition of the recovering channel. Automated control systems and processing of information. Issued by Academy of Civil Aviation, St. Petersburg, Russia, 32-35. (in Russian).

Khoroshavtsev, Y. E. (2005). The conservation of entropy in Markov processes. Scientific News of Belgorod State University. Belgorod. Issue Phys.-Math, 2(22), 132-138. (in Russian).

Khoroshavtsev, Y. E. (2006). Spin in quantum equation. Bulletin of Civil Engineers, 1(6), 99-102 (in Russian).

Khoroshavtsev, Y. E. (2008). The depolarization of spin in the quantum equation. Bulletin of Civil Engineers, 2(15), 101-106 (in Russian).

Kruesi. (2016). Liz.JAXA may remake its X-ray observatory Hitomi for a 2020 launch. Astronomy.com. Retrieved 13.

Landau, L. D., \& Lifshitz, E. M. (1972). The quantum mechanics. Vol.2, Science, Moscow. (in Russian).

Landau, L. D., \& Lifshitz, E. M. (1977). Quantum mechanics: Non - Relativistic theory. Vol. 3 (3 rd, ed).

Mansuripur, M. (1987). Introduction to information theory.

Messiah, A. (1966). Quantum mechanics. Vol. 3. 
Pavlov, G. G., Shibanov, Y. A., \& Gnedin, Y. N. (1979). The influence of polarization on the cyclotron radiation of heat plasma under strong magnetic field. Pis'ma v zhurnal eksperimental'noi i teoreticheskoi fiziki, 30(2), 137-142. (in Russian).

Qi-Ren, Z. (2009) Information conservation, entropy increase and statistical irreversibility for an isolated system. Physic A: Statistical mechanics and applications, 388(19), 4041-4044. https://doi.org/10.1016/j.physa.2009.06.039

Reif, F. (1974). Statistical physics. Berkeley physics course. McGraw - Hill book company. Vol. 5.

Rogerson, J. B., Spitzer, L., Drake, J. F., Dressler, K., Jenkins, E. B., \& Morton, D. C. (1973). Spectrophotometric results from the Copernicus satellite. Instrumentation and performance. Astrophysical Journal, 181. https://doi.org/10.1086/181194

Roncaglia, M. (2017). On the conservation of information in quantum physics. Cornell University, arXiv.org > quant-ph>arXiv: 1708.05727.

Schiff, L. (1949). Quantum mechanics. Maple Press Company, York, PA. https://doi.org/10.1119/1.1989648

Sheridan, T. B., \& Ferrell, W. R. (1975). Man - machine systems: information, control and decision models of human performance. MIT Press. https://doi.org/10.1115/1.3426860

Slichter, C. P. (1992). Principles of magnetic resonance. Berlin, New York. Springer - Verlag.

Sudiarta, I W. (2008). Solving the Schrödinger equation for a charged particle in a magnetic field using the finite difference time domain method. Physics letters A. 372(18), 3145-3148. https://doi.org/10.1016/j.physleta.2008.01.078

Ternov, I. M., \& Mihaylin, V. V. (1986). The cyclotron radiation. The theory and experiment. Energoatomizdat, Moscow, (in Russian).

Wichmann, E. H. (1973). Quantum physics. Berkeley physics course. Vol. 4. McGraw - Hill book company.

\section{Copyrights}

Copyright for this article is retained by the author(s), with first publication rights granted to the journal.

This is an open-access article distributed under the terms and conditions of the Creative Commons Attribution license (http://creativecommons.org/licenses/by/4.0/). 